\title{
SOME THEOREMS ON THE CONVERGENCE OF SERIES.
}

BY PROFESSOR R. D. CARMICHAEL.

(Read before the American Mathematical Society, September 8, 1913.)

No general criteria are known for the study of the convergence of a series of complex terms* when the series does not converge absolutely. The object of this note is to generalize one of the few important special methods which are already available for such study. The theorems obtained are of general applicability; but their practical value lies chiefly in their use in investigating the convergence of series which do not converge absolutely.

In § 1 I give two lemmas which are valuable in themselves; the second is fundamental in the present paper. In $\S 2$ are two general theorems concerning the convergence of series of constant terms, where the convergence need not be absolute. They are generalizations of important results due to Dedekind. In $\$ 3$ two corresponding theorems for uniform convergence are given.

$\S 1$. Statement and Proof of Two Lemmas.-It is convenient to begin with the proof of two lemmas.

LemmA I. Let $s_{1}, s_{2}, s_{3}, \cdots$ be any infinite sequence of numbers having the finite limit s. Then

$$
\lim _{n=\infty} \frac{1}{n^{k+1}} \sum_{i=1}^{n} i^{k} s_{i}=\frac{s}{k+1},
$$

$k$ being a positive integer or zero. $\dagger$

If $\epsilon$ is any positive number there exists a positive integer $t$ such that $\left|s_{i}-s\right|<\epsilon / 2$ for every value of $i$ greater than $t$. For $n>t$, let us write

$$
\frac{1}{n^{k}+1} \sum_{i=1}^{n} i^{k} s_{i}=\frac{1}{n} \sum_{i=1}^{t} \frac{i^{k}}{n^{k}} s_{i}+\frac{1}{n} \sum_{i=t+1}^{n} \frac{i^{k}}{n^{k}} s+\frac{1}{n} \sum_{i=t+1}^{n} \frac{i^{k}}{n^{k}}\left(s_{i}-s\right) .
$$

* The same statement is also true of the special case where the terms are real and are alternately positive and negative.

$\dagger$ For the case when $k=0$ the lemma has been proved by Cesaro, Darboux's Bulletin (2), 14 (1890), pp. 114-120. See Whittaker, Modern Analysis, p. 26. 
Then we have

$$
\begin{aligned}
\left|\frac{1}{n^{k+1}} \sum_{i=1}^{n} i^{k} s_{i}-\frac{1}{n} \sum_{i=t+1}^{n} \frac{i^{k}}{n^{k}} s\right| & \leqq \frac{1}{n} \sum_{i=1}^{t}\left|s_{i}\right|+\frac{1}{n} \sum_{i=t+1}^{n^{\prime}}\left|s_{i}-s\right| \\
& <\frac{t A}{n}+\frac{n-t}{n} \frac{\epsilon}{2}
\end{aligned}
$$

where $A$ is a positive constant such that $\left|s_{i}\right|<A$ for every $i$. It is clear that an $N$ exists such that the last member is less than $\epsilon$ for every $n$ greater than $N$. Therefore it follows at once that we have

Now,

$$
\lim _{n=\infty}\left[\frac{1}{n^{k+1}} \sum_{i=1}^{n} i^{k} s_{i}-\frac{1}{n} \sum_{i=t+1}^{n} \frac{i^{k}}{n^{k}} s\right]=0 .
$$

$$
1^{k}+2^{k}+3^{k}+\cdots+n^{k}=\frac{n^{k+1}}{k+1}+\frac{1}{2} n^{k}+\cdots
$$

the second member being a polynomial in $n$ of degree $k+1$; hence we see readily that

$$
\lim _{n=\infty} \frac{1}{n} \sum_{i=t+1}^{n} \frac{i^{k}}{n^{k}} s=s \lim _{n=\infty} \frac{(t+1)^{k}+(t+2)^{k}+\cdots+n^{k}}{n^{k+1}}=\frac{s}{k+1} .
$$

From this and the preceding limit follows at once the limit given in the conclusion of the lemma.

Corollary. Let $s_{1}, s_{2}, s_{3}, \cdots$ be an infinite sequence of functions of the complex variable $x$ regular in a given closed domain $D$ and converging uniformly to the limit function $s$. Then the limit

$$
\lim _{n=\infty} \frac{1}{n^{k+1}} \sum_{i=1}^{n} i^{k} s_{i}
$$

exists uniformly in $D$ and is $s /(k+1)$.

Certain obvious verbal changes in the proof of the lemma lead readily to the proof of the corollary.

LEMмA II. Let $s_{1}, s_{2}, s_{3}, \cdots$ be any infinite sequence of numbers having the finite limit $s$. Then every solution $u_{n}$ of the difference equation*

$$
\Delta^{k} u_{n}=s_{n}
$$

* We suppose here that a solution is defined only for non-negative integral values of the argument $n$. 
has the property that

$$
\lim _{n=\infty} \frac{u_{n}}{n^{k}}=\frac{s}{k !}
$$

If $\bar{u}_{n}$ is any particular solution of the equation, then the general solution is

$$
u_{n}=\alpha_{0}+\alpha_{1} n+\alpha_{2} n^{2}+\cdots+\alpha_{k-1} n^{k-1}+\bar{u}_{n},
$$

where $\alpha_{0}, \alpha_{1}, \alpha_{2}, \cdots, \alpha_{k-1}$ are independent of $n$. Hence, if the conclusion of the theorem is valid for any particular solution $\bar{u}_{n}$ of the equation, it is valid for every solution. Accordingly we shall prove it for the particular solution defined thus: Write

$$
\Delta^{k-1} \bar{u}_{n}=\sum_{i=1}^{n} s_{i} \equiv n s_{n}^{(1)} \quad(n>0) .
$$

Then, by Lemma I, we see that $\lim _{n=\infty} s_{n}{ }^{(1)}=s$. Then write

$$
\Delta^{k-2} \bar{u}_{n}=\sum_{i=1}^{n} i s_{i}^{(1)} \equiv n^{2} s_{n}^{(2)} \quad(n>0) .
$$

We have $\lim _{n=\infty} s_{n}{ }^{(2)}=s / 2$ !, as we see again by aid of Lemma I. By continuing thus, the proof of the lemma is readily completed.

Corollary. Let $s_{1}, s_{2}, s_{3}, \cdots$ be an infinite sequence of functions of the complex variable $x$ regular in a given closed domain $D$ and converging uniformly to the limit function $s$. Then every solution $u_{n}$ of the difference equation

$$
\Delta^{k} u_{n}=s_{n}
$$

which is regular throughout $D$ has the property that

$$
\lim _{n=\infty} \frac{u_{n}}{n^{k}}
$$

exists uniformly in $D$ and is $s / k !$.

The modifications of the preceding argument, which it is necessary to make to establish the corollary, are obvious.

$\S 2$. Two Theorems on the Convergence of Series of Constant 
Terms.-We are now in position to prove the following theorem:

Theorem I. Let $a_{1}, a_{2}, a_{3}, \cdots$ be any infinite sequence of numbers such that each of the limits*

(1) $\lim _{n=\infty} S_{n}{ }^{(k)}, \lim _{n=\infty} n S_{n}{ }^{(k-1)}, \lim _{n=\infty} n^{2} S_{n}{ }^{(k-2)}, \cdots, \lim _{n=\infty} n^{k-1} S_{n}{ }^{(1)}$ exists and is finite, where

$$
\begin{aligned}
& S_{n}{ }^{(1)}=a_{1}+a_{2}+\cdots+a_{n}, \\
& S_{n}{ }^{(2)}=S_{1}{ }^{(1)}+S_{2}{ }^{(1)}+\cdots+S_{n}^{(1)}, \\
& \cdot \cdot \cdot \cdot \cdot \cdot \cdot \cdot \cdot \cdot \cdot \\
& S_{n}^{(k)}=S_{1}^{(k-1)}+S_{2}{ }^{(k-1)}+\cdots+S_{n}^{(k-1)} .
\end{aligned}
$$

Let $c_{1}, c_{2}, c_{3}, \cdots$ be an infinite sequence of numbers such that

$$
\sum_{i=1}^{\infty}\left|\Delta^{k} c_{i}\right|
$$

is convergent.

Then the series

$$
a_{1} c_{1}+a_{2} c_{2}+a_{3} c_{3}+\cdots
$$

is convergent (but not necessarily absolutely convergent).

It is easy to construct an example to show that the last series is not always absolutely convergent. Hence we have to prove only the affirmative statement in the conclusion of the theorem.

If we adopt the convention that a letter with a negative or a zero subscript has the value zero, we may write $\dagger$

* In case $k=1$ only the first of these limits is to be taken into consideration. The condition on the $a$ 's then is obviously the condition that the series $a_{1}+a_{2}+a_{3}+\cdots$ converges. For this case (namely, when $k=1$ ) the theorem is due to Dedekind (Dirichlet-Dedekind, Zahlentheorie, second edition, 1871 , p. 373). It was rediscovered by Jensen.

$\dagger$ Here we employ repeatedly a fundamental identity due to Abel (Crelle, vol. 1 (1826), p. 314; Oeuvres, 1, p. 222), namely,

where

$$
\sum_{i=1}^{n} v_{i} u_{i} \equiv \sum_{i=1}^{n}\left(V_{i}-V_{i-1}\right) u_{i} \equiv \sum_{i=1}^{n} V_{i}\left(u_{i}-u_{i+1}\right)+V_{n} u_{n+1},
$$

$$
V_{i}=v_{1}+v_{2}+\cdots+v_{i}, \quad i \geqq 1, \quad V_{0}=0 .
$$




$$
\begin{aligned}
S_{n} \equiv \sum_{i=1}^{n} a_{i} c_{i} & =\sum_{i=1}^{n}\left(S_{i}^{(1)}-S_{i-1}^{(1)}\right) c_{i} \\
& =-\sum_{i=1}^{n} S_{i}^{(1)} \Delta c_{i}+S_{n}^{(1)} c_{n+1} \\
& =-\sum_{i=1}^{n}\left(S_{i}^{(2)}-S_{i-1}{ }^{(2)}\right) \Delta c_{i}+S_{n}^{(1)} c_{n+1} \\
& =\sum_{i=1}^{n} S_{i}^{(2)} \Delta^{2} c_{i}-S_{n}{ }^{(2)} \Delta c_{n+1}+S_{n}{ }^{(1)} c_{n+1}
\end{aligned}
$$

An obvious induction proof now leads to the fundamental relation

$$
\begin{aligned}
\text { (3) } S_{n} & =(-1)^{k} \sum_{i=1}^{n} S_{i}^{(k)} \Delta^{k} c_{i}+(-1)^{k-1} S_{n}{ }^{(k)} \Delta^{k-1} c_{n+1} \\
& +(-1)^{k-2} S_{n}{ }^{(k-1)} \Delta^{k-2} c_{n+1}+\cdots-S_{n}{ }^{(2)} \Delta c_{n+1}+S_{n}{ }^{(1)} c_{n+1} .
\end{aligned}
$$

In order to prove our theorem we must show that $S_{n}$ approaches a finite limit as $n$ approaches infinity. To do this, it is sufficient to prove that each term in the second member of (3) approaches a finite limit as $n$ approaches infinity.

That the first term approaches a finite limit follows from the convergence of (2) and the fact that (in consequence of the existence and finite value of the first limit in (1)) a positive constant $A$ exists such that $\left|S_{i}{ }^{(k)}\right|<A$ for every $i$; for then it is clear that the series

converges absolutely.

$$
\sum_{i=1}^{\infty} S_{i}^{(k)} \Delta^{k} c_{i}
$$

Now the series

$$
\sum_{i=1}^{\infty} \Delta^{k} c_{i} \equiv \sum_{i=1}^{\infty}\left(\Delta^{k-1} c_{i+1}-\Delta^{k-1} c_{i}\right)
$$

converges; and hence

$$
\lim _{n=\infty} \Delta^{k-1} c_{n}
$$

exists and is finite. This, in connection with the existence and finite value of the first limit in (1), leads to the conclusion that the second term of the right member of (3) approaches a finite value as $n$ approaches infinity. 
Since $\lim _{n=\infty} \Delta^{k-1} c_{n}$ exists and is finite it follows from Lemma II that each of the limits

$$
\lim _{n=\infty} \frac{\Delta^{k-2} c_{n}}{n}, \lim _{n=\infty} \frac{\Delta^{k-3} c_{n}}{n^{2}}, \cdots, \lim _{n=\infty} \frac{\Delta^{0} c_{n}}{n^{k-1}}
$$

(where $\Delta^{0} c_{n} \equiv c_{n}$ ) exists and is finite. To see this, let us write

$$
u_{n}{ }^{(t)}=\Delta^{k-t} c_{n},
$$

where $t$ is one of the numbers $t=2,3, \cdots, k$. Then

$$
\Delta^{t-1} u_{n}{ }^{(t)}=\Delta^{k-1} c_{n} .
$$

A direct application of the lemma (for each value of $t$ ) now leads to the truth of the statements which we desired to establish.

We retain the same range for the variable $t$ and write

$$
S_{n}{ }^{(k-t+1)} \Delta^{k-t} c_{n+1}=n^{t-1} S_{n}{ }^{(k-t+1)} \cdot \frac{\Delta^{k-t} c_{n+1}}{(n+1)^{t-1}} \cdot \frac{(n+1)^{t-1}}{n^{t-1}}
$$

If $n$ approaches infinity, it is clear (in consequence of (1) and the results just established) that each of the three factors of the second member of (5) approaches a finite limit. From this we see that every term in the right member of (3) past the second approaches a finite limit as $n$ approaches infinity.

This completes the proof of the theorem.

Another valuable theorem may be obtained by making the restrictions on the $c$ 's somewhat stronger and weakening those on the $a$ 's. The special case of this theorem when $k=1$ is also due to Dedekind (1. c., page 371). The general theorem may be stated as follows:

ThEOREM II. Retaining the notation of Theorem I, let us suppose that a positive constant $B$ exists such that each of the quantities

$$
S_{n}{ }^{(k)}, \quad n S_{n}^{(k-1)}, \quad n^{2} S_{n}{ }^{(k-2)}, \quad \cdots, \quad n^{k-1} S_{n}{ }^{(1)}
$$

is in absolute value less than $B$ for every value of $n$. Suppose further that the series

$$
\sum_{i=1}^{\infty}\left|\Delta^{k} c_{i}\right|
$$


is convergent and

Then the series

$$
\lim _{n=\infty} \Delta^{k-1} c_{n}=0 .
$$

$$
a_{1} c_{1}+a_{2} c_{2}+a_{3} c_{3}+\cdots
$$

is convergent (but not necessarily absolutely convergent).

As before, we have the fundamental relation (3). It is sufficient to show that each term in the second member of (3) again approaches a finite limit. The first term may be dealt with precisely as in the preceding argument. The second term approaches zero, as one sees directly from the hypothesis of the theorem. Since $\lim _{n=\infty} \Delta^{k-1} c_{n}=0$ it follows from Lemma II that each of the limits in (4) is zero. Hence, from (5) it follows that every term in the right member of (3) after the second approaches zero as $n$ approaches infinity. This completes the proof of the theorem.

As an example illustrative of the theory, let us consider the series

where

$$
\sum_{n=1}^{\infty}(-1)^{n-1} \frac{2 n+1}{n(n+1)} c_{n}(\rho) \quad(\rho>0),
$$

$$
c_{n}(\rho)=1+\frac{1}{2^{\rho}}+\frac{1}{3^{\rho}}+\cdots+\frac{1}{n^{\rho}} .
$$

Let the constant $c_{n}$ of theorem II be $c_{n}(\rho)$. Then

$$
\Delta c_{n}=\frac{1}{(n+1)^{\rho}}, \quad \Delta^{2} c_{n}=\frac{1}{(n+2)^{\rho}}-\frac{1}{(n+1)^{\rho}}=\frac{-\mu}{n^{\rho+1}}+\cdots ;
$$

whence it follows that $\Sigma\left|\Delta^{2} c_{i}\right|$ converges and $\lim _{n=\infty} \Delta c_{n}=0$. Thus $c_{n}$ satisfies the conditions requisite for applying Theorem II when $k=2$.

Put

$$
a_{1}=\frac{1}{2}, \quad a_{n}=(-1)^{n-1} \frac{2 n+1}{n(n+1)} \quad(n>1) .
$$

Then series (6) is identical with $a_{1} c_{1}+a_{2} c_{2}+a_{3} c_{3}+\cdots$ except for the first term; hence it is sufficient to prove the convergence of the last series. We have 


$$
\begin{aligned}
S_{1}^{(1)}=\frac{\mathrm{I}}{2}, \quad S_{n}^{(1)}= & \frac{1}{2}-\frac{5}{2 \cdot 3}+\frac{7}{3 \cdot 4}-\cdots+(-1)^{n-1} \frac{2 n+1}{n(n+1)} \\
= & \frac{1}{2}-\left(\frac{1}{2}+\frac{1}{3}\right)+\left(\frac{1}{3}+\frac{1}{4}\right) \\
& \quad-\cdots+(-1)^{n-1}\left(\frac{1}{n}+\frac{1}{n+1}\right) \\
= & (-1)^{n-1} \frac{1}{n+1} \quad(n>1) ; \\
S_{n}{ }^{(2)}= & \frac{1}{2}-\frac{1}{3}+\cdots+(-1)^{n-1} \frac{1}{n+1} .
\end{aligned}
$$

It is clear that $S_{n}{ }^{(1)}$ and $S_{n}{ }^{(2)}$ satisfy the conditions requisite for the application of Theorem II.

Hence we conclude the convergence of series (6).

\$3. Two Theorems on Uniform Convergence.-By employing the corollaries to Lemmas I and II instead of these lemmas themselves and making certain obvious verbal changes in the proofs as given in $\S 2$, it is easy to establish the following two theorems:

TheOREM III. Let $a_{1}, a_{2}, a_{3}, \cdots$ be any infinite sequence of numbers such that each of the limits

$$
\lim _{n=\infty} S_{n}{ }^{(k)}, \quad \lim _{n=\infty} n S_{n}{ }^{(k-1)}, \quad \lim _{n=\infty} n^{2} S_{n}{ }^{(k-2)}, \cdots, \lim _{n=\infty} n^{k-1} S_{n}{ }^{(1)}
$$

exists and is finite, where $S_{n}{ }^{(1)}, \cdots, S_{n}{ }^{(k)}$ have the same meaning as in Theorem $I$. Let $c_{1}, c_{2}, c_{3}, \cdots$ be an infinite sequence of functions of the complex variable $x$ regular in a given closed domain $D$ and such that

$$
\sum_{i=1}^{\infty}\left|\Delta^{k} c_{i}\right|
$$

converges uniformly in $D$.

Then the series

$$
a_{1} c_{1}+a_{2} c_{2}+a_{3} c_{3}+\cdots
$$

converges uniformly in $D$.

THEOREM IV. Retaining the same notation as in Theorem III, let us suppose that a positive constant $B$ exists such that each of the quantities 


$$
S_{n}{ }^{(k)}, \quad n S_{n}{ }^{(k-1)}, \quad n^{2} S_{n}^{(k-2)}, \quad \cdots, \quad n^{k-1} S_{n}{ }^{(1)}
$$

is in absolute value less than $B$ for every value of $n$. Suppose further that the series

$$
\sum_{i=1}^{\infty}\left|\Delta^{k} c_{i}\right|
$$

converges uniformly in $D$ and

Then the series

$$
\lim _{n=\infty} \Delta^{k-1} c_{n}=0 \text {. }
$$

$$
a_{1} c_{1}+a_{2} c_{2}+a_{3} c_{3}+\cdots
$$

converges uniformly in $D$.

For the case $k=1$ both of these theorems are already known. This special case of Theorem III appears to have been first employed by Nielsen*; but Nielsen's statement of it is not entirely accurate, as Landau $\dagger$ has pointed out. The corresponding special case of Theorem IV is due to Cahen.

INDIANA UNIVERSITY, July, 1913.

\title{
A TRANSLATION PRINCIPLE CONNECTING THE INVARIANT THEORY OF LINE CONGRUENCES WITH THAT OF PLANE $n$-LINES.
}

\author{
BY PROFESSOR O. E. GLENN.
}

(Read before the American Mathematical Society, September 9, 1913.)

A weLL known translation principle of Clebsch§ enables us to construct a ternary invariant by a simple algebraical operation upon a binary invariant. In brief, if the line $u_{x}=0$ intersects the curve

$$
f=a_{x}{ }^{n}=b_{x}{ }^{n}=\cdots=0
$$

in the $n$ points given by the binary form

* Annali di Matematica (3), 15 (1908), pp. 275-282.

† Sitzungsber. d. K. Bayer. Akad. d. Wiss., Phys.-Math. Classe, 1909.

$\ddagger$ Annales scientifiques de l'Ecole Normale Supérieure (3), 11 (1894), p. 79.

$\S$ Clebsch, Vorlesungen über Geometrie, vol. 1, p. 276; and Journal für Math., vol. 59 (1861). 